\title{
Flozins - in light of the latest recommendations
}

\author{
Marta Wróbel, Dominika Rokicka, Krzysztof Strojek \\ Department of Internal Medicine, Diabetology, and Cardiometabolic Disorders, Faculty of Medical Sciences Zabrze, \\ Medical University of Silesia in Katowice, Zabrze, Poland
}

\begin{abstract}
$\mathrm{F}$ ozins (SGLT-2 inhibitors) have been used in the treatment of diabetes since 2012. These drugs were initially approved for the treatment of type 2 diabetes as monotherapy if metformin was contraindicated or not tolerated, or in combination with other drugs and insulin if the therapy was intensified. Often in Polish conditions, the role of flozins was reduced to their use as a third-line drug added to metformin and a sulfonylurea. This was a recent attempt to intensify oral therapy in patients with type 2 diabetes who were not adequately compensated before the decision to switch to insulin. Recent years have shown additional benefits resulting from the use of flozins. This resulted in a change in the position of this group of drugs (ex aequo with metformin as first-line) in the type 2 diabetes treatment regimen in the recommendations of many recognized diabetes societies, including the Polish Diabetes Society. In addition, these drugs have strengthened their position in the treatment of diseases other than diabetes.
\end{abstract}

\section{Anti-hyperglycaemic and cardio-renal activity of flozins}

Flozins do not affect the action of insulin, so they do not generate hypoglycaemia. By blocking the SGLT-2 receptor in the distal tubule of the nephron, they reduce glucose reuptake and thus increase its excretion in the urine, resulting in a desired reduction in blood glucose levels. Thanks to flozins, as much as $80 \mathrm{~g}$ of glucose, i.e. about $300 \mathrm{kcal}$ per day, is lost, which translates into a reduction in body weight of about $2.5-3 \mathrm{~kg}$ in some patients. Blockade of the SGLT-2 receptor also reduces sodium and chlorine reabsorption in the proximal tubule, which leads to an increased influx of sodium and chlorine into the macula densa. As a result, the concentration of adenosine increases, the afferent arterioles contract, and the glomerular pressure is reduced. Flozins additionally reduce both the intra-renal activity of the RAA system and the sympathetic system, which are compensatively increased in the course of heart failure. The natriuretic effect and osmotic diuresis translate into a reduction in blood pressure and a reduction in the preload and hence afterload of the heart, which is beneficial for both the kidneys and the failure of the heart muscle [1].

\section{Flozins in clinical trials}

The presence of cardiovascular complications multiplies the risk of death in the diabetic population. The need to assess cardiovascular safety before the registration of new glycaemia-lowering drugs forced the pharmaceutical industry to conduct studies assessing cardiovascular points such as the risk of cardiovascular death, heart attack, stroke, or hospitalization for cardiovascular reasons. The first study to demonstrate the cardiological benefits of flozins was the EMPA-REG OUTCOME study, in which empagliflozin was added to the standard of care for type 2 diabetes in patients with cardiovascular disease. This study showed that empagliflozin treatment resulted in a 38\% reduction in the risk of cardiovascular death, an effect independent of the degree of glycaemic control $\left(\mathrm{HbA}_{1 \mathrm{c}} \mathrm{ap}-\right.$ proximately $8 \%$ in both groups) [2]. Both the EMPA-REG OUTCOME study and the subsequent studies: CANVAS with canagliflozin and DECLARE-TIMI 58 with dapagliflozin, conducted in the population of patients with type 2 diabetes with established cardiovascular disease and risk factors for its development, showed not only the beneficial effect of adding these preparations to standard antihyperglycaemic treatment to control diabetes, but also to reduce the risk of hospitalization due to heart failure $[3,4]$. Similar results confirming the beneficial effect of flozins in heart failure were shown in studies which included patients with and without diabetes diagnosed with symptomatic heart failure (NYHA II-IV) — DAPA HF (study with dapagliflozin) and patients with kidney damage — CREDENCE 
(study with canagliflozin) [5,6]. Based on the results of the above-mentioned studies, the reduction in hospitalization for heart failure was estimated at approximately $30 \%$ in patients using one of the three listed flozins. The results of the EMPEROR-Reduced study, which confirmed the efficacy of empagliflozin in the population of patients with heart failure and reduced left ventricular ejection fraction, both with and without diabetes, were highly significant [7]. Similar results were obtained in the DAPA-HF study for dapagliflozin. On this basis, the indications for the use of both flozins were extended in patients with heart failure without accompanying diabetes.

The range of therapeutic options in patients with heart failure with lowered left ventricular ejection fraction is wide. Treatment is limited in patients with heart failure and preserved left ventricular ejection fraction $(\mathrm{EF}>40 \%)$. In the EMPEROR-PRESERVED study, the addition of empagliflozin to existing therapy in heart failure patients with preserved ejection fraction was associated with a $21 \%$ reduction in the risk of cardiovascular death or hospitalization for heart failure (HR: 0.79; $\mathrm{p}<0.001$ ) [8]. The results of this study suggest that the protective effect of flozins on the heart is independent of the degree of heart failure. Recently published results from the EMPA-Heart study showed that six-month use of empagliflozin in patients with type 2 diabetes and coronary heart disease was associated with a decreased left ventricular extracellular volume (ECV), suggesting reversibility of myocardial remodelling [9]. Heart failure leads to worsening of, among others, kidney function. The occurrence of renal failure in patients with heart failure is estimated at $25 \%$. It has been known for years that the initiation of therapy with flozins is associated with an initial decrease in the GFR value, which is a functional phenomenon and the most beneficial one because it indicates a decrease in intraglomerular pressure. Over time, the reduction in glomerular hyperfiltration and the reduction in intraglomerular pressure, especially in the early stages of diabetes, contributes to the maintenance of renal function [10]. The first randomized study to confirm the nephroprotective effect of flozins was the CREDENCE study with canagliflozin, which showed that the drug in patients with diabetes and chronic kidney disease (patients with GFR $>30 \mathrm{~mL} / \mathrm{min} / 1.73 \mathrm{~m}^{2}$ were recruited) significantly reduced the risk of serious cardiovascular and renal events [6]. In the DAPA-CKD study with dapagliflozin, the patient population, both with and without diabetes (treated with an angiotensin converting enzyme inhibitor [ACEI] or angiotensin receptor blockers [ARB]) was mainly patients with impaired renal function (up to GFR $25 \mathrm{~mL} / \mathrm{min} / 1.73 \mathrm{~m}^{2}$ ). Dapagliflozin has been shown to reduce the risk of developing a major kidney point (50\% decline in GFR or end-stage renal disease) and the risk of renal and cardiovascular death [11]. The results of these studies represent a breakthrough in the treatment of patients with chronic kidney disease because until recently the only drugs that improve the function of the kidneys were thought to be ACEI and ARB.

\section{Recommendations}

The results of these studies were the basis for changes in the recommendations of many scientific societies, including the change in the recommendations of the European Society of Cardiology (ESC) in 2019, which included flozins and GLP-1 analogues, instead of metformin, as the first-choice drugs in the treatment of patients with newly diagnosed type 2 diabetes and cardiovascular disease or with high cardiovascular risk. The ESC proposes metformin as a first-line treatment only in overweight patients, without cardiovascular disease, or with a moderate risk of cardiovascular disease. At the same time, it should be borne in mind that a large proportion of patients with type 2 diabetes are people at high cardiovascular risk. In previous years, the recommendations of this society were consistent with those of diabetes societies, and metformin was considered the first-line drug in the treatment of newly diagnosed type 2 diabetes. The controversial position of the ESC has not been confirmed in clinical trials and is in contradiction with the recommendations of diabetes societies, for which metformin is still a first-line drug [12]. According to the joint statement of the American Diabetes Association and the European Association for the Study of Diabetes contained in the 2019 updated consensus on the treatment of hyperglycaemia in the course of type 2 diabetes in patients at high risk of developing cardiovascular complications, the addition of flozin or a GLP-1 agonist should be considered, regardless of the HbA1c value. Due to the reduction of hospitalization for heart failure, major cardiovascular events, and deaths from cardiovascular causes, SGLT-2 inhibitors are particularly recommended as initial therapy in combination with metformin in the presence of heart failure, especially with a reduced ejection fraction $\left(\mathrm{EF}<45 \%\right.$ ) and chronic kidney disease (eGFR 30 to $\leq 60 \mathrm{~mL} / \mathrm{min} / 1.73 \mathrm{~m}{ }^{2}$ or ACR [albumin/creatinine ratio] $>30 \mathrm{mg} / \mathrm{g}$, in particular ACR $>300 \mathrm{mg} / \mathrm{g}$ ), regardless of baseline $\mathrm{HbA}_{1 \mathrm{c}}$ or individualized $\mathrm{HbA1c}$ target [13]. The 2021 recommendations of the Polish Diabetes Society also advise the initiation of antihyperglycemic therapy in patients with newly diagnosed type 2 diabetes and systolic heart failure or chronic kidney disease immediately from combining the SGLT-2 inhibitor with metformin as the first-line treatment. In a patient previously treated with metformin, it is necessary to add flozin if, in addition to diabetes mellitus, the above conditions coexist [14]. The benefits of the cardioprotective effect of flozins also translated into the addition 
of this group of drugs to the pool of recognized cardiological preparations used in the treatment of heart failure in patients with or without diabetes. In accordance with the latest ESC recommendations from 2021, in the section devoted to the treatment of heart failure, the initiation of therapy in heart failure with impaired left ventricular systolic function should be started with five drugs simultaneously, among which, in addition to ACEI/ ARNI inhibitors (angiotensin-converting enzyme inhibitor; angiotensin receptor-neprilysin inhibitor), beta-blockers, mineralocorticoid receptor antagonists, and loop diuretics, are flozins (dapagliflozin or empagliflozin) (class of recommendation IA). Canagliflozin, dapagliflozin, empagliflozin, ertugliflozin, and sotagliflozin (not available in Poland) are recommended in patients with type 2 diabetes and a high risk of cardiovascular events, to reduce hospitalization for heart failure, major cardiovascular events, end-stage renal disease, and death for cardiovascular reasons (class IA). Dapagliflozin, empagliflozin, and sotagliflozin are recommended in patients with type 2 diabetes and heart failure with impaired ejection fraction to reduce hospitalization for heart failure and cardiovascular death [15]. The beneficial effect of SGLT-2 inhibitors on the cardiovascular system and kidneys was also reflected in the recommendations of KIDGO (Kidney Disease Improving Global Outcomes) [16]. These drugs are recommended for patients with type 2 diabetes and chronic kidney disease with GFR 30-60 mL/min/1.73 $\mathrm{m}^{2}$ (class IA).

The indications for dapagliflozin and empagliflozin (SmPC) have been extended. In addition to the treatment of type 2 diabetes (and at a dose of $5 \mathrm{mg}$ also as an adjunct to therapy in type 1 diabetes), dapagliflozin is indicated for chronic symptomatic heart failure with reduced left ventricular ejection fraction and for the treatment of chronic kidney disease in patients with and without diabetes. Due to the protective effect on the kidneys, this drug can be used up to a GFR of $25 \mathrm{~mL} / \mathrm{min} / 1.73 \mathrm{~m}^{2}$, without the need to reduce the dose. One of the indications for empagliflozin therapy is, in addition to type 2 diabetes, heart failure with a reduced left ventricular ejection fraction with a GFR value of not less than $20 \mathrm{~mL} / \mathrm{min} / 1.73 \mathrm{~m}^{2}$. It is crucial to be aware that the antihyperglycemic effect of flozins in patients with severe renal impairment is weakened because a small amount of glucose is then filtered and excreted by the kidneys. In such a situation, only their non-diabetic effects should be expected.

\section{Summary}

Patients with type 2 diabetes are at risk of developing serious complications, mainly cardiovascular and renal. Therefore, the used antidiabetic preparations should not only safely lower glycaemia but also have a beneficial effect on other organs. Flozins fit perfectly with the above characteristics, because their therapeutic potential has already exceeded the treatment of diabetes.

\section{References}

1. Sieradzki J. Cukrzyca. Via Medica, Gdańsk 2019.

2. Zinman B, Wanner C, Lachin JM, et al. EMPA-REG OUTCOME Investigators. Empagliflozin, Cardiovascular Outcomes, and Mortality in Type 2 Diabetes. N Engl J Med. 2015; 373(22): 2117-2128, doi: 10.1056/NEJMoa1504720, indexed in Pubmed: 26378978.

3. Wiviott S, Raz I, Bonaca M, et al. Dapagliflozin and Cardiovascular Outcomes in Type 2 Diabetes. New Engl J Med. 2019; 380(4): 347-357, doi: 10.1056/nejmoa1812389, indexed in Pubmed: 30415602.

4. Neal B, Perkovic V, Mahaffey KW, et al. CANVAS Program Collaborative Group. Canagliflozin and Cardiovascular and Renal Events in Type 2 Diabetes. N Engl J Med. 2017; 377(7): 644-657, doi: 10.1056/NEJMoa1611925, indexed in Pubmed: 28605608.

5. McMurray JJV, Solomon SD, Inzucchi SE, et al. Dapagliflozin in patients with heart failure and reduced ejection fraction. N Engl J Med. 2019; 381(21): 1995-2008, doi: 10.1056/NEJMoa1911303, indexed in Pubmed: 31535829.

6. Perkovic V, Jardine MJ, Neal B, et al. CREDENCE Trial Investigators. Canagliflozin and Renal Outcomes in Type 2 Diabetes and Nephropathy. N Engl J Med. 2019; 380(24): 2295-2306, doi: 10.1056/NEJMoa1811744, indexed in Pubmed: 30990260

7. Packer M, Butler J, Filippatos G, et al. Evaluation of the effect of sodium-glucose co transporter 2 inhibition with empagliflozin on morbidity and mortality of patients with chronic heart failure and a reduced ejection fraction: rationale for and design of the EMPEROR Reduced trial. Eur J Heart Fail. 2019; 21(10): 1270-1278, doi: 10.1002/ejhf.1536, indexed in Pubmed: 31584231.

8. Anker SD, Butler J, Filippatos G, et al. EMPEROR-Preserved Trial Investigators. Empagliflozin in Heart Failure with a Preserved Ejection Fraction. N Engl J Med. 2021 [Epub ahead of print], doi: 10.1056/NEJMoa2107038, indexed in Pubmed: 34449189.

9. Mason T, Coelho-Filho O, Verma S, et al. Empagliflozin Reduces Myocardial Extracellular Volume in Patients With Type 2 Diabetes and Coronary Artery Disease. JACC: Cardiovasc Imag. 2021; 14(6): 1164-1173, doi: 10.1016/j.jcmg.2020.10.017, indexed in Pubmed: 33454272.

10. Nespoux J, Vallon V. SGLT2 inhibition and kidney protection. Clin Sci (Lond). 2018; 132(12): 1329-1339, doi: 10.1042/CS20171298, indexed in Pubmed: 29954951.

11. Heerspink HJL, Stefansson BV, Chertow GM, et al. DAPA-CKD Investigators. Rationale and protocol of the Dapagliflozin And Prevention of Adverse outcomes in Chronic Kidney Disease (DAPA-CKD) randomized controlled trial. Nephrol Dial Transplant. 2020; 35(2): 274-282, doi: 10.1093/ndt/gfz290, indexed in Pubmed: 32030417.

12. Cosentino F, Grant PJ, Aboyans V, et al. ESC Scientific Document Group. 2019 ESC Guidelines on diabetes, pre-diabetes, and cardiovascular diseases developed in collaboration with the EASD. Eur Heart J. 2020; 41(2): 255-323, doi: 10.1093/eurheartj/ehz486, indexed in Pubmed: 31497854.

13. Buse JB, Wexler DJ, Tsapas A, et al. 2019 Update to: Management of Hyperglycemia in Type 2 Diabetes, 2018. A Consensus Report by the American Diabetes Association (ADA) and the European Association for the Study of Diabetes (EASD). Diabetes Care. 2020; 43(2): 487-493, doi: 10.2337/dci19-0066, indexed in Pubmed: 31857443.

14. Araszkiewicz A, Bandurska-Stankiewicz E, Borys S, et al. 2021 Guidelines on the management of patients with diabetes. A position of Diabetes Poland. Clin Diabetol. 2021; 10(1), doi: 10.5603/DK.2021.0001.

15. Seferović P, Coats A, Ponikowski P, et al. European Society of Cardiology/Heart Failure Association position paper on the role and safety of new glucose lowering drugs in patients with heart failure. Eur J Heart Fail. 2020; 22(2): 196-213, doi: 10.1002/ejhf.1673, indexed in Pubmed: 31816162.

16. Karagiannis T, Tsapas A, Bekiari E. KDIGO made 12 recommendations for managing diabetes with CKD. Ann Int Med. 2021; 174(3): JC26, doi: 10.7326/acpj202103160-026, indexed in Pubmed: 33646846. 